\title{
Mit Erfahrung und integrativem Blick zur sicheren Diagnose!
}

\author{
Bei akuten Thoraxschmerzen gibt es nicht den einen Befund oder den einen Biomarker. Die \\ Differenzialdiagnose ist eine Herausforderung. EKG-Veränderungen treten etwa treten auch bei \\ Lungenembolie, Aortensyndrom oder Pneumothorax auf.
}

\begin{abstract}
Akute Thoraxschmerzen können vielfältige Ursachen haben. Oft würden die Beschwerden durch lebensbedrohliche Erkrankungen wie Lungenembolie, Aortitis, Herzinfarkt oder Aortendissektion verursacht, sodass rasches und richtiges Handeln erforderlich sei, betonte Dr. Matthias Held, Pneumologe am Würzburger Zentrum für pulmonale Hypertonie und Lungengefäßkrankheiten. Die Differentialdiagnose ist allerdings nicht immer einfach, wenn keine CT-Angiografie verfügbar ist. „Es gibt nicht den einen Befund oder den einen Biomarker für die sichere Diagnose, es braucht vielmehr klinische Erfahrung und den integrativen Blick“, so Held.

Es gebe reihenweise Patienten mit akuten Thoraxschmerzen, bei denen zunächst ein NSTEMI-Infarkt vermutet werde, die aber was anderes haben. Zu den möglichen pneumologischen Ursachen zählt außer einer Lungenembolie zum Beispiel auch ein Lungenhochdruck oder ein (Spannungs-)Pneumothorax.

Laut Daten des Würzburger Lungenembolie-Registers war nur bei $60 \%$ der Patienten die Verdachtsdiagnose bei der Klinikeinlieferung richtig und immerhin jeder dritte gab als Symptom auch Thoraxschmerzen an. Die Symptome von Lungenembolie-Patienten seien unspezifisch und unterschieden sich häufig kaum bei Patienten mit bestätigter oder nicht bestätigter Lungenembolie, so Held.
\end{abstract}

\section{EKG-Veränderungen auch bei anderen Erkrankungen}

Natürlich helfe ein EKG bei der Differentialdiagnose, führt der Pneumologe aus, aber EKG-Veränderungen gebe es nicht selten auch bei Lungenembolie, Aortensyndrom oder Pneumo- thorax. Bei Lungenembolie-Patienten könnten zum Beispiel TNegativierungen über der Vorderwand auftreten und bei rund $5 \%$ der Patienten mit Aortensyndrom seien ST-Hebungen beschrieben. Bei Patienten mit Pneumothorax zeigt sich im EKG nicht selten eine Abnahme der QRS-Amplitude, ein linksseitiger Pneumothorax kann auch ischämietypische Veränderungen verursachen. $\mathrm{Zu}$ bedenken sei auch, dass eine Embolie zu Vorhofflimmern (VHF) führen könne, sagte Held. Der umgekehrte Zusammenhang ist ebenfalls bekannt: VHF-Patienten haben ein erhöhtes Risiko für Lungenembolien.

\section{Biomarker sind meist nicht spezifisch genug}

Auch Biomarker ermöglichen keine sichere Diagnose. Erhöhte Troponin-Spiegel seien nicht nur typisch für einen Myokardschaden (Herzinfarkt, Herzinsuffizienz), sondern auch bei Patienten mit Lungenembolie, pulmonaler Hypertonie oder Aortendissektion nachweisbar, so Held. Und ein positiver D-DimerTest sei nur bei $95 \%$ der Patienten spezifisch für eine Lungenembolie. Ein weit verbreiteter Irrglaube ist nach Angaben des Pneumologen, dass Patienten mit vorbestehender Antikoagulation keine Lungenembolie haben könnten. Nach Daten des Würzburger Registers erhielten immerhin 6\% der Lungenembolie-Patienten Antikoagulanzien; sie waren aber meist schlecht eingestellt oder kürzlich umgestellt worden.

„Wir brauchen zur Differentialdiagnose bei Patienten mit akuten Thoraxschmerzen die Bildgebung", resümierte der Pneumologe, idealerweise eine CT-Angiografie.

Roland Fath
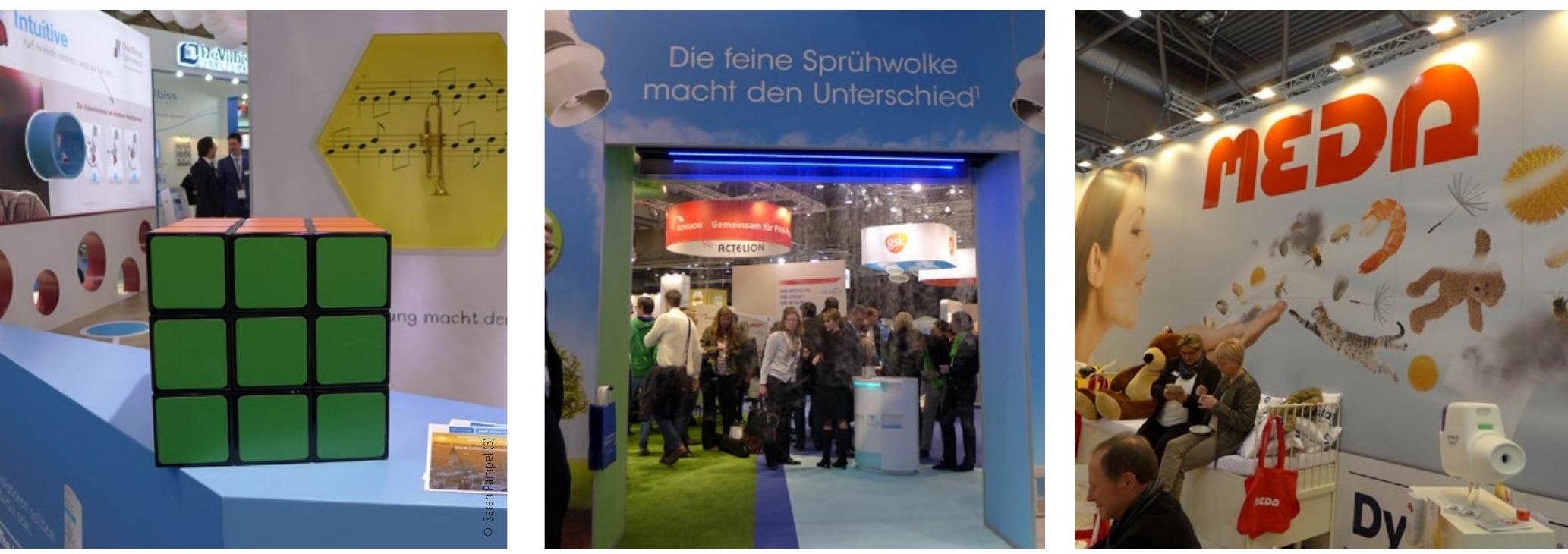

...das Fachpublikum fand hier auch alte Bekannte vor, etwa den Zauberwürfel, ebenso wie eine umfangreiche Industrieaustellung. 